\title{
PULSED AND UNPULSED GAMMA-RAY EMISSION FROM MILLISECOND PULSARS
}

\author{
D. M. WeI, ${ }^{1,2}$ K. S. CHENG, ${ }^{1}$ AND T. $\mathrm{LU}^{2,3}$ \\ Received 1995 July 25; accepted 1996 March 15
}

\begin{abstract}
We use the outer gap model to study the gamma-ray emission and pair production processes of millisecond pulsars. The small light cylinder of millisecond pulsars results in a large optical depth for gamma rays produced inside the light cylinder. Electron/position cascade will take place until the energy of emitted gamma rays is less than a specific threshold energy $\left(E_{\text {crit }}\right)$. The model for the pulsed gamma-ray spectrum is $F_{1}\left(E_{\gamma}\right) \sim E_{\gamma}^{-2}$ for $E_{\gamma} \leq E_{\text {crit }}$. Furthermore, electrons/positrons accelerated in the outer gap can continue to interact with the low-energy gamma rays beyond the light cylinder to produce very high energy gamma rays. The subsequent pair production and radiation processes can produce unpulsed high-energy photons in the energy range of the EGRET detector on board the Compton Gamma Ray Observatory with a model spectrum $F_{2}\left(E_{\gamma}\right) \sim E_{\gamma}^{-3 / 2}$ for most millisecond pulsars. We have calculated the theoretical spectra of PSR J0751+18, PSR 1821-24, and PSR 1937+21 to illustrate our model. Unpulsed gamma-ray emission from other sources, e.g., 47 Tuc, are discussed also.
\end{abstract}

Subject headings: gamma rays: theory - pulsars: general - radiation mechanisms: nonthermal

\section{INTRODUCTION}

Lundgren, Zepka, \& Cordes (1993) have reported the discovery of a 3.5 ms binary pulsar, PSR J0751+18, with an orbital period of $6.3 \mathrm{hr}$ in the error box of the EGRET source GRO J0752+17. This high-energy source was detected at the $4.6 \sigma$ level (Hartman et al. 1992) during an early viewing period. Sturner \& Dermer (1994) have applied four pulsar gamma-ray emission models with certain assumptions to the millisecond pulsar and find that if the $3.5 \mathrm{~ms}$ pulsar is indeed the source of the gamma-ray emission, its emission will either be unpulsed or this millisecond pulsar cannot have been recycled according to current recycling models (Bhattacharya \& van den Heuvel 1991). The period derivative $\dot{P}$, and thus the inferred magnetic field of this pulsar, has been measured recently (Lundgren, Zepka, \& Cordes 1995). The spin-down power of this pulsar is less than the observed upper limit of the gamma-ray luminosity. In fact, the published version of the gamma-ray catalog of EGRET did not include this source (Fichtel et al. 1993). Nevertheless, gamma rays from a millisecond pulsar provide a very interesting topic for study.

It is generally assumed that the pulsed radiation comes from the pulsar and unpulsed gamma-ray emission from the nebula. Gould (1965) has proposed a Comptonsynchrotron model to account for the steady emission from the nebula. He suggested that the energetic electrons in the nebula can produce photons (IR-X-rays) through synchrotron radiation, and these same photons may be boosted up to high-energy photons by inverse Compton scattering with the energetic electrons. This model was developed further by Rieke \& Weekes (1969) and Grindlay \& Hoffman (1971). On the other hand, by using the outer magnetospheric gap model (Cheng, Ho, \& Ruderman 1986a, 1986b; Ho 1989), which was proposed to explain the pulsed gamma-ray emission from the Crab pulsar, an alternative mechanism was proposed to explain the unpulsed high-energy gamma-ray

\footnotetext{
${ }^{1}$ Department of Physics, University of Hong Kong, Pokfulam Road, Hong Kong.

2 Department of Astronomy, Nanjing University, Nanjing, 210093, China.

${ }^{3}$ CCAST (World Laboratory), P.O. Box 8730, Beijing, 100080, China.
}

emission from the Crab pulsar (Kwok, Cheng, \& Lau 1991; Cheung \& Cheng 1993). Here we extend this model to the millisecond pulsars and calculate the unpulsed gamma-ray emission spectrum from millisecond pulsars.

This paper is organized as follows: In $\S 2$ we review some key results of the outer gap model and the model of unpulsed very high energy (VHE) gamma-rays from the Crab pulsar. In $\S 3$, we first estimate the pulsed gamma-ray spectrum emitted from regions inside the light cylinder. Then the production mechanisms and the processes of unpulsed gamma rays beyond the light cylinder are presented as well. In $\S 4$, the detailed calculation of the unpulsed gamma-ray spectrum is given. In $\S 5$, we discuss the highenergy emission from globular clusters. Finally, we show the numerical model results and give a brief conclusion.

\section{OUTER GAP MODEL AND UNPULSED VHE GAMMA-RAY EMISSION MODEL}

Cheng et al. (1986a, 1986b, hereafter CHR I and CHR II) constructed an outer magnetospheric gap model of rapidly spinning neutron stars. They assume that global current flow in the outer magnetosphere results in a large region of magnetospheric charge depletion (gap) in which $\boldsymbol{E} \cdot \boldsymbol{B} \neq 0$. This gap acts as an ultrarelativistic particle accelerator, which can generate a large number of $e^{ \pm}$pairs to limit their size and to short out $\boldsymbol{E} \cdot \boldsymbol{B}$. The process is as follows: In the Crab-type gap, the gap primary electrons and positrons lose most of their energy through curvature radiation, and their energy is limited by radiation reaction. The curvature gamma rays (gap primary gamma rays) convert to $e^{ \pm}$pairs (gap secondary pairs) in collision with gap X-rays, which come from the synchrotron radiation of the gap secondary pairs. Inverse Compton scattering of the gap secondary pairs on these gap X-rays boosts a significant fraction of gap X-rays to gamma rays. The combination of the synchrotron spectrum and inverse Compton scattering spectrum is proposed to explain the observed pulsed emission of the Crab pulsar from IR to gamma rays.

Beyond the light cylinder, where the radius of curvature is much larger than $10^{8} \mathrm{~cm}$, the curvature radiation becomes unimportant, and the gap primary $e^{ \pm}$pairs may 


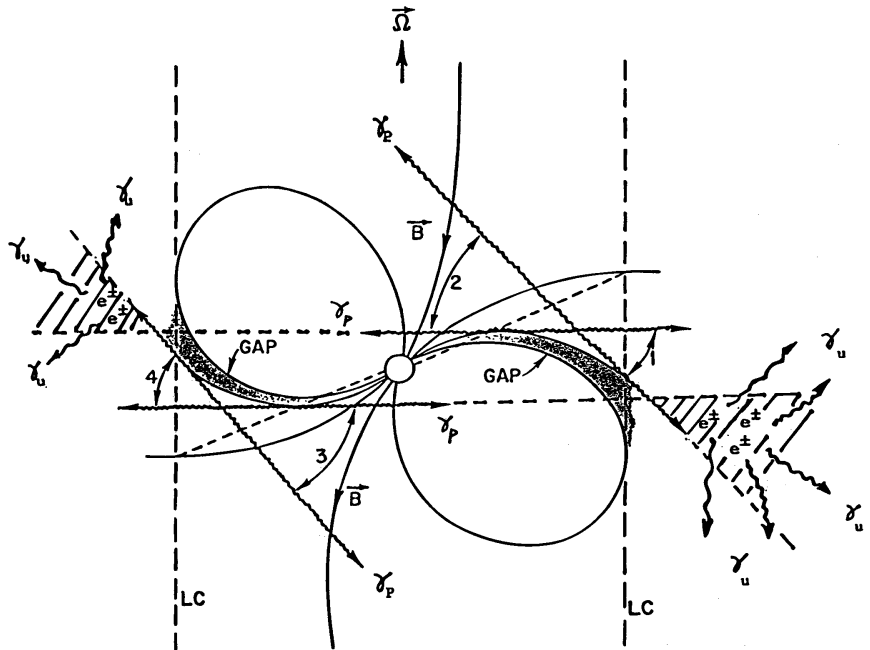

FIG. 1.-Schematic representation of the pair production and radiation processes beyond the light cylinder (LC), where the corotation speed equals the speed of light. The shaded regions are the locations of the outer gap in which gap primary electrons and positrons are accelerated and lose their energies to high-energy curvature photons. These curvature gamma rays (gap primary photons) convert to gap secondary $e^{ \pm}$pairs in collisions with X-rays which come from the synchrotron emission from the same gap secondary pairs created beyond the outer gap. Four pulsed gamma-ray beams $\left(\gamma_{p}\right)$ are emitted by these gap secondary pairs via synchrotron radiation and inverse Compton scattering processes. Beyond the light cylinder, beam 1 (2) and beam 3 (4) will cross over each other (hatched regions) and result in secondary $e^{ \pm}$pairs with large pitch angles. The subsequent radiation of these secondary pairs results in unpulsed gamma rays $\left(\gamma_{u}\right)$ in the energy ranges of $\mathrm{X}$-ray to $\mathrm{TeV}$ gamma ray.

maintain their energies. It is also interesting to note that the gap secondary photons have very little chance to collide with the gap primary pairs. This is because the $e^{ \pm}$pairs are generally constrained to move relativistically along the curved magnetic field lines. The photons they emit are then radiated mainly into a narrow cone whose axis is parallel to the local $\boldsymbol{B}$. Such photons always propagate into the region above the curved $\boldsymbol{B}$ (on its convex side) but generally not into the regions below (on the concave side). However, the gap primary $e^{ \pm}$pairs from one outer gap will inevitably collide with the gap IR-optical photons from the other outer gap and produce TeV primary VHE gamma rays and vice versa (see Fig. 1). The primary VHE gamma rays are sufficiently energetic to produce secondary $e^{ \pm}$pairs by collision with the same pulsed IR-optical photons, since the mean free path of conversion processes is large enough to result in nearly isotropic secondary gamma rays (Kwok et al. 1991; Cheung \& Cheng 1993).

\section{THE PRODUCTION MECHANISMS OF UNPULSED GAMMA RAYS FROM MILLISECOND PULSARS}

Before presenting the unpulsed gamma-ray emission model for millisecond pulsars, it should be noted that the optical depth for the collision between two beams of gap secondary photons (i.e., beam 1 and 2 or beam 3 and 4 in Fig. 1) is large, since the radius of the light cylinder, $r_{l}$, is very small for millisecond pulsars. As an example, we can use the gap secondary photon spectrum of the millisecond pulsar PSR 1821-24 calculated by the synchrotron selfCompton model, which is proposed to account for the $\mathrm{X}$-ray and gamma-ray emission from pulsars (Cheng \& Wei
1995), to estimate the optical depth of collision between two crossing beams of gap secondary photons:

$$
\tau_{\gamma} \simeq \frac{F_{\gamma}}{\Delta \Omega r_{l}^{2} c} \sigma_{p} r_{l},
$$

where $F_{\gamma}\left(\right.$ photons s $\left.^{-1}\right) \sim 10^{43}$ photons s $^{-1}$ with $E_{\gamma} \geq 1 \mathrm{keV}$ (see eqs. [4.8] and [4.13]) is the calculated gap secondary photon flux, $r_{l} \sim 10^{7} \mathrm{~cm}, \sigma_{p}\left(\sim \sigma_{\mathrm{T}} / 3\right)$ is the maximum cross section of pair production, and $\Delta \Omega(\sim 1 \mathrm{sr})$ is the solid angle of the gap secondary photon beam. We can see that $\tau_{\gamma}>1$ for a photon with energy $E_{\gamma}>\left(2 m c^{2}\right)^{2} / 1 \mathrm{keV} \simeq 1 \mathrm{GeV}$. Hence, we believe that in millisecond pulsars the pulsed high-energy photons cannot escape from the pulsars until their energy is below a threshold $\left(E_{\text {crit }}\right)$, defined when the optical depth of $\tau\left(E_{\text {crit }}\right)=1$ (see eq. [4.9]). Since the collision between gap secondary photons can produce the gap tertiary $e^{ \pm}$pairs, these $e^{ \pm}$pairs may continue to produce the gap tertiary photons through synchrotron radiation or inverse Compton scattering. Therefore, we may argue that the photon index of the gap photon spectrum is about -2 for $E_{\gamma}<E_{\text {crit }}$ (this argument is consistent with the numerical results presented in Cheng \& Wei 1995).

In this paper, we assume that the unpulsed gamma rays come from a compact region, a couple of light cylinder radii beyond the pulsar rather than from the extended nebula $\langle r\rangle$, since the gap primary electrons/positrons from one gap and the IR photons from another gap are expected to cross over just beyond the light cylinder (see Fig. 1). The process of producing the unpulsed gamma-ray emission is as follows: When the energetic gap primary $e^{ \pm}$pairs pass through the light cylinder, they will collide with the pulsed low-energy photons emitted from the other gap at distance $r>r_{l}$, where $r_{l}=c / \Omega$ is the radius of the light cylinder. The low-energy photons will be boosted up to about $1 \mathrm{TeV}$ through inverse Compton scattering. These primary energetic photons $\left[F_{1}\left(E_{\gamma}\right)\right]$ will collide again with the same pulsed low-energy photons and then produce the secondary $e^{ \pm}$pairs. The secondary pairs may produce the observed photons by either synchrotron radiation or inverse Compton scattering. So it is obvious the observed gamma rays should contain three components: (1) the surviving primary photons $F_{1}\left(E_{\gamma}\right) e^{-\tau\left(E_{\gamma}\right)},(2)$ the flux of synchrotron radiation $F_{2}\left(E_{\gamma}\right)$, and (3) the flux from inverse Compton scattering $F_{3}\left(E_{\gamma}\right)$.

Qualitatively, we could argue that these gamma rays produced beyond the light cylinder should be emitted isotropically. There are two main reasons. First, the magnetic field inside the light cylinder has a dipolar form, but it will gradually transform to a swirling magnetic field (see Fig. 2 of Kwok et al. 1991) beyond the light cylinder. The gap secondary photons are emitted tangentially to the local magnetic field lines inside the light cylinder, but the secondary pairs created by collisions with the gap X-rays can make arbitrary pitch angles with the local magnetic field lines beyond the light cylinder because the locations at which photons are emitted and at which they materialize to pairs are not necessarily correlated. Second, the mean free path of the "unpulsed" $0.1 \mathrm{TeV}$ gamma rays can be estimated as

$$
\lambda \sim\left[n(\langle r\rangle) \sigma_{p}\right]^{-1},
$$

where $n \approx F_{\gamma}(10 \mathrm{eV}) / 4 \pi\langle r\rangle^{2} c \approx 10^{18} \mathrm{~cm}^{-3}$ is the tertiary photon density of PSR $1821-24$ at the mean distance $\langle r\rangle$ of the pair production, taken to be $3 r_{l}$. We find that $\lambda \sim r_{l}$, 
which implies that the average pitch angle of secondary pairs is of order unity, so the emission from these secondary $e^{ \pm}$pairs is nearly isotropic.

\section{CALCULATION OF THE UNPULSED GAMMA-RAY SPECTRUM}

According to the model of CHR I and CHR II, the primary $e^{ \pm}$pairs inside the outer gap are accelerated to extreme relativistic energies. The total potential of the outer gap can be expressed as

$$
\Delta V=6 \times 10^{12} f^{2} B_{12} P^{-2} \mathrm{~V},
$$

and the rate of particles passing through the gap is

$$
\dot{N}=2.5 \times 10^{30} f B_{12} P^{-2} \mathrm{~s}^{-1},
$$

where $P$ is the period of the pulsar, $B_{12}$ is the surface magnetic field in units of $10^{12} \mathrm{G}$, and $f$ is the fractional volume of the outer magnetosphere occupied by the outer gap. If every pulsar has the same angle between the magnetic axis and the rotation axis, $f$ can be scaled from that of the Crab pulsar (Cheng \& Wei 1995):

$$
f \simeq 0.1\left(\frac{B}{B_{\text {Crab }}}\right)^{-39 / 60}\left(\frac{P}{P_{\text {Crab }}}\right)^{99 / 60}
$$

However, for Crab-type pulsars, the primary $e^{ \pm}$pairs accelerated inside the outer gap cannot achieve the energy of the full potential of the gap because they will lose energy by curvature radiation. The maximum energy of the primary $e^{ \pm}$pair is

$$
\gamma_{\max } \simeq\left(\frac{\Delta V}{e} s\right)^{1 / 4},
$$

where $s$ is the curvature radius of magnetic field lines. Hence, the energy distribution of the gap primary $e^{ \pm}$pairs is monoenergetic and can be expressed as

$$
\frac{d \dot{N}_{e}}{d E_{e}}=\dot{N} \delta\left(E_{e}-\gamma_{\max } m c^{2}\right) .
$$

Cheng \& Ding (1994) have concluded that the Crab-type pulsar should have a period

$$
P \leq 5 \times 10^{-2} B_{12}^{2 / 5} \mathrm{~s},
$$

which is obtained by equating the efficiencies of Crab-type and Vela-type pulsars. But Chen \& Ruderman (1993) have pointed out that the inclination angle may play a crucial role in pulsar evolution, so a pulsar with period larger than that given in equation (4.6) may still be Crab-type as long as its inclination angle is larger than that of the Crab pulsar. So, we assume that equation (4.3) can be written as

$$
f \simeq \begin{cases}f_{\text {Crab }}=0.066 B_{9}^{-0.65}(P / 1 \mathrm{~ms})^{1.65} & \\ 1 & \text { if } f_{\text {Crab }}>1 .\end{cases}
$$

We choose the millisecond pulsar whose value of $f$ is close to that of the Crab pulsar as a candidate for emission of gamma rays, since we believe that the millisecond pulsars should be similar to the Crab pulsar in many ways. From the pulsar catalog, we find that PSR 1821-24 and PSR $1937+21$ are the best candidates because their value of $f$ is 0.25 (equation [4.3]) and their periods are less than that given in equation (4.6). As for the millisecond pulsar
$\mathrm{J} 0751+18$, its parameters yield $f_{\text {Crab }} \simeq 1.4$, which implies that this cannot be a Crab-like pulsar unless its inclination angle is larger than that of the Crab pulsar. Observationally, if this is a gamma-ray pulsar, it will be expected to emit double pulses with $180^{\circ}$ separation (Cheng \& Ding 1994). To illustrate our model, we will assume $f \simeq 1$ for PSR $\mathrm{J} 0751+18$

The primary gamma-ray spectrum $d^{2} N_{p}\left(E_{\gamma}\right) / d E_{\gamma} d t$ is produced through inverse Compton scattering of gap primary electrons/positrons by the gap tertiary photons emitted from the other gap. It has been argued in the last section that the photon index of the gap gamma-ray spectrum is about -2 , so we assume that the incident photon spectrum has the form

$$
\frac{d \dot{N}}{d E}=A_{1} E^{-2}, \quad E_{\min } \leq E \leq E_{\text {crit }},
$$

where $E_{\text {crit }}$ is defined when the optical depth equals unity, namely,

$$
A_{1}\left[\frac{\left(2 m c^{2}\right)^{2}}{E_{\text {crit }}}\right]^{-1} \sigma_{p} / \Delta \Omega r_{l} c \sim 1,
$$

and $E_{\min }=\max \left[\hbar(e B / m c), h v_{c}\right], v_{c}$ is the synchrotron selfabsorption frequency which can be given by

$$
v_{c} \simeq 10^{4}\left[3.56 \frac{e \Delta V \dot{N}}{r_{l} c \Delta \Omega m c^{2}}(B f)^{5 / 2}\right]^{2 / 7} \mathrm{~Hz},
$$

and when $E_{\min }=h v_{c}$, we have

$$
\frac{d \dot{N}}{d E}=B E^{-3 / 2}, \quad E<h v_{c},
$$

or if $E_{\min }=\hbar(e B / m c)$, we have

$$
\frac{d \dot{N}}{d E}=B E^{-2 / 3}, \quad E<\hbar \frac{e B}{m c} .
$$

For typical millisecond pulsars $\left(B_{9}=1\right.$ and $\left.P=3 \mathrm{~ms}\right)$, the synchrotron self-absorption frequency and the cyclotron frequency are $10^{14} \mathrm{~Hz}$ and $2 \times 10^{13} \mathrm{~Hz}$, respectively. On the other hand, according to the conservation of energy, we may have

$$
e \Delta V \dot{N}=\int E \frac{d \dot{N}}{d E} d E .
$$

From equations (4.8)-(4.13) we can obtain the coefficients $A_{1}, A_{2}$, and $E_{\text {crit }}$; then the incident photon spectrum is completely determined. Using equation (4.5), we can obtain the primary gamma-ray spectrum (Blumenthal \& Gould 1970),

$$
\begin{aligned}
\frac{d^{2} N_{p}\left(E_{\gamma}\right)}{d E_{\gamma} d t}= & N_{0} F\left(E_{\gamma}, \gamma_{\max }\right) \\
= & N_{0} \frac{3 \sigma_{\mathrm{T}} c}{4 \gamma_{\max }^{2}} \int_{0}^{\infty} \frac{n(\epsilon)}{\epsilon}[2 q \ln q+(1+2 q)(1-q) \\
& \left.+\frac{1}{2} \frac{(\Gamma q)^{2}(1-q)}{1+\Gamma q}\right] d \epsilon
\end{aligned}
$$


where $\quad \Gamma=4 \epsilon \gamma_{\max } / m c^{2}, \quad q=E_{1} /\left[\Gamma\left(1-E_{1}\right)\right], \quad E_{1}=$ $E_{\gamma} / \gamma_{\max } m c^{2}$, and $n(\epsilon)=1 / \Delta \Omega\langle r\rangle^{2} c d \dot{N} / d \epsilon$. Here $\sigma_{\mathrm{T}}$ is the Thomson cross section; $N_{0}=\dot{N} \phi\langle r\rangle / c$ is the average number of primary $e^{ \pm}$pairs inside the region of inverse Compton scattering; $\phi$ is the average azimuthal width of the gap gamma-ray beam; $m$ is the electron mass; and $c$ is the speed of light.

Some of these primary photons will be attenuated by the same incident photons to produce the secondary $e^{ \pm}$pairs. The observed primary photon spectrum $F_{1}\left(E_{\gamma}\right)$ has been modified to

$$
F_{1}\left(E_{\gamma}\right)=\frac{d^{2} N_{p}\left(E_{\gamma}\right)}{d E_{\gamma} d t} e^{-\tau\left(E_{\gamma}\right)}
$$

where $\tau\left(E_{\gamma}\right)$ is the attenuation depth due to pair production. Here $\tau\left(\mathrm{E}_{\gamma}\right)$ is given by (Jauch \& Rohrlich 1976)

$$
\tau\left(E_{\gamma}\right)=\langle r\rangle \int_{\epsilon_{\min }}^{\infty} n(\epsilon) \sigma d \epsilon,
$$

where $\sigma=\sigma_{\mathrm{T}}(3 / 16)\left(1-v^{2}\right)\left\{\left(3-v^{4}\right) \ln [(1+v) /(1-v)]-2 v\right.$ $\left.\times\left(2-v^{2}\right)\right\}, v=\left[1-\left(m^{2} c^{4} / E_{\gamma} \epsilon\right)\right]^{1 / 2}$, and $\epsilon_{\min }=m^{2} c^{4} / E_{\gamma}$. Here we have assumed that two photons are in a head-on collision.

These secondary $e^{ \pm}$pairs will produce the unpulsed secondary gamma rays through synchrotron radiation or inverse Compton scattering. Before we find the secondary photon spectrum, let us calculate first the energy distribution $d N\left(E_{e}\right) / d E_{e}$ of the secondary pairs with energy $E_{e}$. It is given by (Blumenthal \& Gould 1970)

$$
\frac{d N\left(E_{e}\right)}{d E_{e}}=\frac{1}{\dot{E}_{e}} \int_{E_{e}}^{E_{\max }} \dot{Q}\left(E_{e}^{\prime}\right) d E_{e}^{\prime},
$$

where $E_{\max }=\gamma_{\max } m c^{2} / 2, \quad \dot{Q}\left(E_{e}^{\prime}\right)=2\left(1-e^{-\tau\left(E_{e}^{\prime}\right)}\right)\left[d^{2} N_{p}\left(E_{e}^{\prime}\right) /\right.$ $\left.d E_{e}^{\prime} d t\right], \quad \tau\left(E_{e}^{\prime}\right)=\tau\left(E_{\gamma}=2 E_{e}^{\prime}\right), \quad$ and $\quad d^{2} N_{p}\left(E_{e}^{\prime}\right) / d E_{e}^{\prime} d t=$ $d^{2} N_{p}\left(E_{\gamma}=2 E_{e}^{\prime}\right) / d E_{\gamma} d t$ is given by equation (4.14). The total energy loss of secondary $e^{ \pm}$pairs is $\dot{E}_{e}=\left(\dot{E}_{e}\right)_{\mathrm{syn}}+\left(\dot{E}_{e}\right)_{\mathrm{ICS}}$, where the energy loss due to synchrotron radiation is

$$
\left(\dot{E}_{e}\right)_{\mathrm{syn}}=\frac{\sigma_{\mathrm{T}} c E_{e}^{2} B^{2}}{4 \pi m^{2} c^{4}},
$$

with

$$
B \simeq B_{s}\left(\frac{R}{r_{l}}\right)^{3}\left(\frac{r_{l}}{\langle r\rangle}\right),
$$

and the energy loss due to inverse Compton scattering is

$$
\left(\dot{E}_{e}\right)_{\mathrm{ICS}}=\frac{3}{8} \sigma_{\mathrm{T}} m^{2} c^{5} \int_{0}^{\infty} \frac{n(\epsilon)}{\epsilon}\left[\ln \left(\frac{4 \epsilon E_{e}}{m^{2} c^{4}}\right)-\frac{11}{6}\right] d \epsilon .
$$

Once the spectrum of the secondary pairs is found, the secondary gamma-ray spectrum of synchrotron radiation is given by

$$
F_{2}\left(E_{\gamma}\right)=\int \frac{d N\left(E_{e}\right)}{d E_{e}}\left[\frac{3^{1 / 2} e^{3} B}{h m c^{2}} \frac{1}{E_{c}\left(E_{e}\right)} \int_{E_{\gamma /} / E_{c}}^{\infty} K_{5 / 3}(\xi) d \xi\right] d E_{e},
$$

where $E_{c}\left(E_{e}\right)=3 h e B E_{e}^{2} /\left(4 \pi m^{3} c^{5}\right)$ is the characteristic energy, $h$ is the Planck constant, $e$ is the electronic charge, and $K_{5 / 3}(\xi)$ is the modified Bessel function. The secondary gamma-ray spectrum from inverse Compton scattering is

$$
F_{3}\left(E_{\gamma}\right)=\int \frac{d N\left(E_{e}\right)}{d E_{e}} F\left(E_{\gamma}, E_{e}\right) d E_{e},
$$

where $F\left(E_{\gamma}, E_{e}\right)$ is given by equation (4.14) with $\gamma_{\max }$ replaced by $E_{e} / m c^{2}$.

It should be noted that the unpulsed gamma rays thus produced are only the first generation of unpulsed gamma rays. In principle, these photons will be absorbed further at a mean distance $\langle r\rangle_{i}$ from the pulsar and produce the next generation of the unpulsed gamma rays. The further pair production processes are essentially the same as those of the first generation of pair production, with the difference in the following input parameters:

$$
\begin{gathered}
\langle r\rangle_{i}=\langle r\rangle_{i-1}+\lambda_{i-1} \text { for } i>1, \\
\dot{Q}_{i}\left(E^{\prime}\right)=2\left(1-e^{-\tau\left(E_{\gamma}\right)}\right) F_{i-1}\left(E_{e}^{\prime}=E_{\gamma} / 2\right) \text { for } i>1,
\end{gathered}
$$

where the subscript $i$ denotes the $i$ th generation of unpulsed gamma rays, $\lambda_{i-1}$ is the mean free path of the $(i-1)$ th generation gamma rays, and $F_{i-1}$ is the unpulsed gammaray spectrum of the $(i-1)$ th generation.

Thus, the total observed unpulsed gamma-ray spectrum $F_{\text {tot }}$ is the sum of all the generations of the surviving unpulsed gamma-ray spectrum:

$$
F_{\mathrm{tot}}\left(E_{\gamma}\right)=\sum_{i=1}^{\infty} F_{i}\left(E_{\gamma}\right) e^{-\tau_{i}\left(E_{\gamma}\right)} .
$$
is

Finally, the unpulsed gamma-ray flux observed on Earth

$$
F_{\text {obs }}\left(E_{\gamma}\right)=\frac{F_{\text {tot }}\left(E_{\gamma}\right)}{4 \pi D^{2}},
$$

where $D$ is the distance to the pulsar.

\section{HIGH-ENERGY EMISSION FROM GLOBULAR CLUSTERS}

Up to now, many millisecond pulsars have been discovered in several globular clusters. For example, 11 have been found in 47 Tucanae (Manchester et al. 1991). A large number of millisecond pulsars are expected in globular cluster cores in which the formation rate of accreting binary systems is high, and some models predict that the total number of millisecond pulsars in some globular clusters are in the range of hundreds or thousands (Kulkarni, Narayan, \& Romani 1990; Wijers \& Van Paradijs 1991; Tavani 1993). So it is very interesting to discuss the high-energy emission from globular clusters. According to our model, these millisecond pulsars will contribute to the high-energy emission of globular clusters. Therefore, using the observed gamma-ray flux from globular clusters, we can give the upper limits on the number of millisecond pulsars inside a globular cluster.

We would like to emphasize that in our model there are two types of gamma-ray spectra: one is pulsed and the 
other is unpulsed. In order to calculate the gamma-ray emission from globular clusters, in the following we estimate the pulsed and unpulsed gamma-ray spectra analty:cally rather than numerically as before.

The pulsed photons are emitted inside the light cylinder, and their expected spectrum is

$$
F_{\gamma}=\frac{d^{2} N_{\gamma}}{d E_{\gamma} d t}=A E_{\gamma}^{-2}, \quad E_{\min } \leq E_{\gamma} \leq 2 m c^{2},
$$

where

$$
A=P_{1} / \log \left(\frac{2 m c^{2}}{E_{\min }}\right),
$$

with $\log \left(2 m c^{2} / E_{\min }\right) \simeq 20$, and

$$
P_{1}=\dot{N} e \Delta V,
$$

in which $\dot{N}, \Delta V$ are given by equations (4.1)-(4.3), we have

$$
P_{1}=7 \times 10^{33} B_{9}^{1 / 20}(P / 1 \mathrm{~ms})^{19 / 20} \mathrm{ergs}^{-1} .
$$

On the other hand, the unpulsed components are those photons radiated outside the light cylinder, and their spectrum can be estimated in the following way. It is not difficult to see that the energy loss rate due to synchrotron radiation is larger than that of inverse Compton scattering beyond the light cylinder at $\langle r\rangle \sim 3 r_{l}$. This is because

$$
\frac{P_{\text {syn }}}{P_{\text {ICS }}} \simeq \frac{B^{2}(\langle r\rangle) / 8 \pi}{P_{1} / 4 \pi\langle r\rangle^{2} c} \gg 1 \text {. }
$$

In other words, the $e^{ \pm}$pairs created beyond the light cylinder will lose their energies mainly through synchrotron radiation.

The primary VHE gamma-ray spectrum $d^{2} N_{\mathrm{ICS}} / d E_{\gamma} d t$ is produced through inverse Compton scattering between the gap primary $e^{ \pm}$pairs and the gap low-energy photons emitted from the other gap outside the light cylinder. The calculation shows that the inverse Compton scattering spectrum is very flat,

$$
\frac{d^{2} N_{\mathrm{ICS}}}{d E_{\gamma} d t} \propto \text { constant }, \quad E_{\gamma} \leq \frac{\gamma_{\max } m c^{2}}{2}
$$

where

$$
\gamma_{\max }=2.6 \times 10^{7} s_{7}^{1 / 4} f^{1 / 2} B_{9}^{1 / 4}\left(\frac{P}{1 \mathrm{~ms}}\right)^{-1 / 2}
$$

is the Lorentz factor of primary $e^{ \pm}$pairs. The energy spectrum of the secondary electron/positron pairs is

$$
\frac{d N_{e}}{d E_{e}}=\frac{1}{\dot{E}_{e}} \int_{E_{e}}^{E_{\max }} \dot{Q}\left(E_{e}^{\prime}\right) d E_{e}^{\prime},
$$

where $E_{\max }=\frac{1}{2} \gamma_{\max } m c^{2} / 2$. Since $\dot{E}_{e} \propto E_{e}^{2}$ for synchrotron radiation, we obtain

$$
\frac{d N_{e}}{d E_{e}} \propto E_{e}^{-2}\left(E_{\max }-E_{e}\right) \sim E_{e}^{-2}, \quad E_{e} \leq E_{\max } .
$$

Therefore, the secondary unpulsed photon spectrum emitted through synchrotron radiation of secondary $e^{ \pm}$ pairs is

$$
\frac{d^{2} N_{\gamma}}{d E_{\gamma} d t} \propto E_{\gamma}^{-(2+1) / 2} \sim E_{\gamma}^{-3 / 2}, \quad E_{\gamma} \leq\left(E_{\gamma}\right)_{\max },
$$

where

$$
\begin{aligned}
\left(E_{\gamma}\right)_{\max } & =\frac{3}{2}\left(\frac{E_{\max }}{m c^{2}}\right)^{2} \hbar \frac{e B(\langle r\rangle)}{m c} \\
& =1.4 \times 10^{11} B_{9}^{17 / 20}\left(\frac{P}{1 \mathrm{~ms}}\right)^{-141 / 60} s_{7}^{1 / 2} \mathrm{eV},
\end{aligned}
$$

with $B(\langle r\rangle)$ given by equation (4.19).

Hence, these unpulsed high-energy gamma rays should have a photon spectrum

$$
F_{\gamma}\left(E_{\gamma}\right) \simeq A^{\prime} E_{\gamma}^{-3 / 2}, \quad E_{\gamma} \leq\left(E_{\gamma}\right)_{\max }
$$

where

$$
A^{\prime}=\frac{1}{2} P_{2}\left(E_{\gamma}\right)_{\max }^{-1 / 2},
$$

and

$$
P_{2}=\dot{N} \gamma_{\max } m c^{2}=10^{33} B_{9}^{11 / 40} s_{7}^{1 / 4}\left(\frac{P}{1 \mathrm{~ms}}\right)^{-1 / 40} \mathrm{ergs}^{-1},
$$

or roughly

$$
P_{2} \simeq 10^{33} B_{9}^{11 / 40} \mathrm{~s}_{7}^{1 / 4} \mathrm{ergs}^{-1} .
$$

Here we have assumed that most of the outgoing kinetic energy in primary gap particles will be converted into the unpulsed photon energy. This is justified because the optical depths for both inverse Compton scattering and $\gamma-\gamma$ pair production near the light cylinder are greater than unity.

It should be noted again that the pulsed component is in the low-energy range $\left(E_{\gamma} \leq E_{\text {crit }}\right)$, so it can only be detected by OSSE. On the other hand, the unpulsed component is in the energy range of EGRET. Therefore, we may get the limits on the number of millisecond pulsars inside a globular cluster according to the observed pulsed or unpulsed gamma-ray flux detected by OSSE or EGRET.

For the pulsed component, the average photon flux in the energy range of OSSE is

$$
\begin{aligned}
\langle F\rangle= & \frac{A \int_{0.3 \mathrm{MeV}}^{1} E_{\gamma}^{-2} d E_{\gamma}}{4 \pi d^{2}}=3.3 \times 10^{-7} B_{9}^{1 / 20} \\
& \times\left(\frac{P}{1 \mathrm{~ms}}\right)^{19 / 20}\left(\frac{d}{3 \mathrm{kpc}}\right)^{-2} \mathrm{~cm}^{-2} \mathrm{~s}^{-1},
\end{aligned}
$$

and the sensitivity of OSSE in the MeV energy range is about $3 \times 10^{-4} \mathrm{~cm}^{-2} \mathrm{~s}^{-1}$. Here we can define a critical number

$$
N_{1}=\frac{3 \times 10^{-4}}{\langle F\rangle} \simeq 10^{3} B_{9}^{-1 / 20}\left(\frac{P}{1 \mathrm{~ms}}\right)^{-19 / 20}\left(\frac{d}{3 \mathrm{kpc}}\right)^{2} .
$$

So, if OSSE did not detect gamma-ray flux from a globular cluster, we may conclude that the number of millisecond pulsars inside this globular cluster, $N$, should be less than $N_{1}$, approximately $N<10^{3}$.

Similarly, the average photon flux of the unpulsed component is

$$
\begin{aligned}
\langle F\rangle= & \frac{A^{\prime} \int_{100 \mathrm{MeV}}^{\left(E_{\gamma}\right)_{\max }} E_{\gamma}^{-3 / 2} d E_{\gamma}}{4 \pi d^{2}} \simeq 2 \times 10^{-10} B_{9}^{-3 / 20} \\
& \times\left(\frac{P}{1 \mathrm{~ms}}\right)^{23 / 20}\left(\frac{d}{3 \mathrm{kpc}}\right)^{-2} \mathrm{~cm}^{-2} \mathrm{~s}^{-1}
\end{aligned}
$$


and the sensitivity of EGRET is about $7 \times 10^{-8} \mathrm{~cm}^{-2} \mathrm{~s}^{-1}$. Again, we can also define another critical number

$$
N_{2}=\frac{7 \times 10^{-8}}{\langle F\rangle}=3.5 \times 10^{2} B_{9}^{3 / 20}\left(\frac{P}{1 \mathrm{~ms}}\right)^{-23 / 20}\left(\frac{d}{3 \mathrm{kpc}}\right)^{2}
$$

thus, if EGRET did not detect unpulsed gamma-ray emission from a globular cluster, it means that the number of millisecond pulsars inside this globular cluster should be less than $N_{2}$; in general, $N<400$.

Recently, Michelson et al. (1994) reported the upper limits to the high-energy gamma-ray emission from millisecond pulsars in a number of globular clusters, including 47 Tuc. They find that there is no strong evidence for highenergy gamma-ray emission from any of these clusters. Therefore, according to the above discussion, we may conclude that the number of millisecond pulsars in these globular clusters should be less than 400 ; this may constrain the formation rate of millisecond pulsars in globular clusters.

\section{CALCULATED RESULTS AND CONCLUSION}

In calculating the unpulsed gamma-ray spectrum, we need first to specify the two parameters $\langle r\rangle$ and $\langle\phi\rangle$. These two parameters depend on the angle between the rotation axis and the magnetic axis, and on the exact field structure near the light cylinder, neither of which is known. However, $\langle\phi\rangle$ should be less than $2 \pi$ and $\langle r\rangle$ around a few light cylinder radii because the pair production processes must take place first near the light cylinder. In our numerical calculations, $\langle\phi\rangle$ and $\langle r\rangle$ are chosen to be the same as those of the Crab pulsar (Cheung \& Cheng 1993), namely, 1 rad and $3 r_{l}$, respectively.

Figures 2 and 3 show the calculated pulsed and unpulsed gamma-ray spectrum of pulsar PSR 1821-24 and PSR $1937+21$. For the unpulsed spectrum, the solid line, dotted line, and dot-dashed line represent the total flux, flux of surviving primary inverse Compton spectrum, and flux of the secondary inverse Compton spectrum, respectively. The arrow indicates the sensitivity of EGRET. It shows that the theoretical value is about 2 orders of magnitude less than

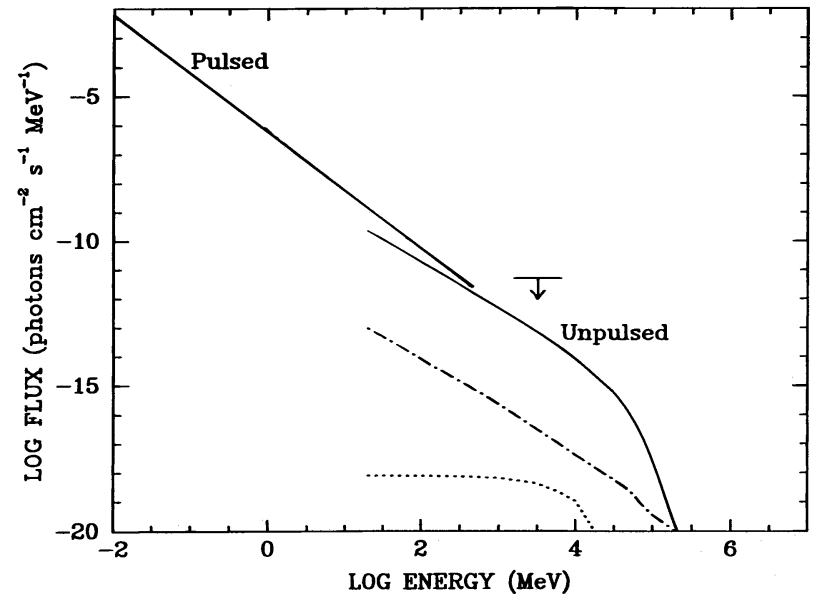

FIG. 2.-The calculated pulsed and unpulsed gamma-ray spectrum of PSR 1821-24. For the unpulsed spectrum, the solid line is the total flux, the dotted line is the surviving primary inverse Compton spectrum, and the dot-dashed line is the flux of secondary inverse Compton scattering. The arrow indicates the sensitivity of EGRET. The pulsed component cutoff is at $E_{\text {crit }} \sim 0.5 \mathrm{GeV}$.

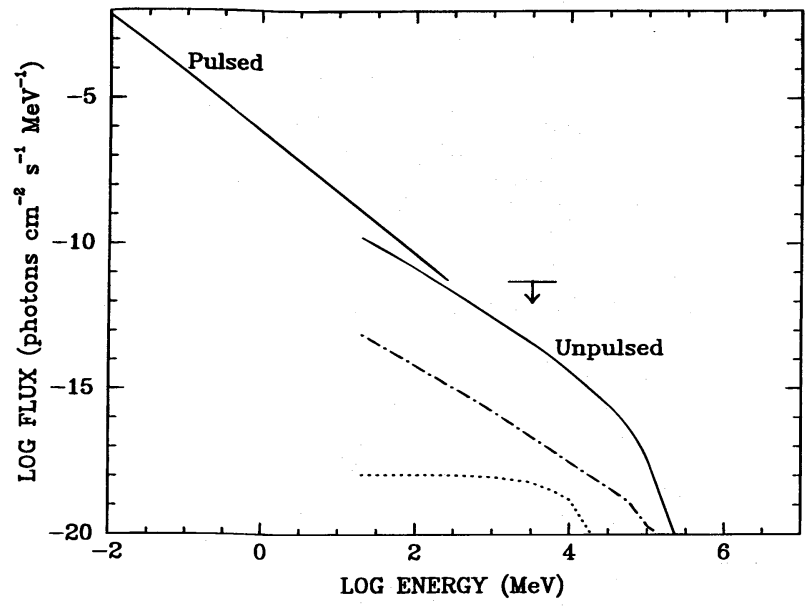

FIG. 3.-Same as Fig. 2, except for PSR $1937+21$ and $E_{\text {crit }} \sim 0.2 \mathrm{GeV}$

the sensitivity of EGRET; therefore, we conclude that if our model is correct, then it is very difficult to detect the highenergy gamma rays emitted from these two pulsars.

Figure 4 shows the calculated pulsed and unpulsed gamma-ray spectrum of millisecond pulsar PSR J0751 + 18 . The arrow symbol represents the gamma-ray flux upper limit detected by EGRET. It shows that the theoretical result is below the observed upper limit by a factor of several, so we suggest that this $3.5 \mathrm{~ms}$ pulsar may be a possible EGRET source in future when more data have been accumulated.

In summary, in this paper we assume that the unpulsed high-energy gamma-ray emission comes from a compact region, a couple of light cylinder radii beyond the millisecond pulsar. We have compared our model results for three millisecond pulsars. In addition, we have also discussed the pulsed and unpulsed gamma-ray emission from globular clusters, and we can set upper limits on the number of millisecond pulsars in a globular cluster by comparing with the observations.

This work is partially supported by an RGC grant of Hong Kong and a CRCG grant from the University of Hong Kong. We thank the referee for pointing out the recent paper by Lundgen, Zepka, \& Cordes and his useful suggestions and T. C. Boyce for a critical reading.

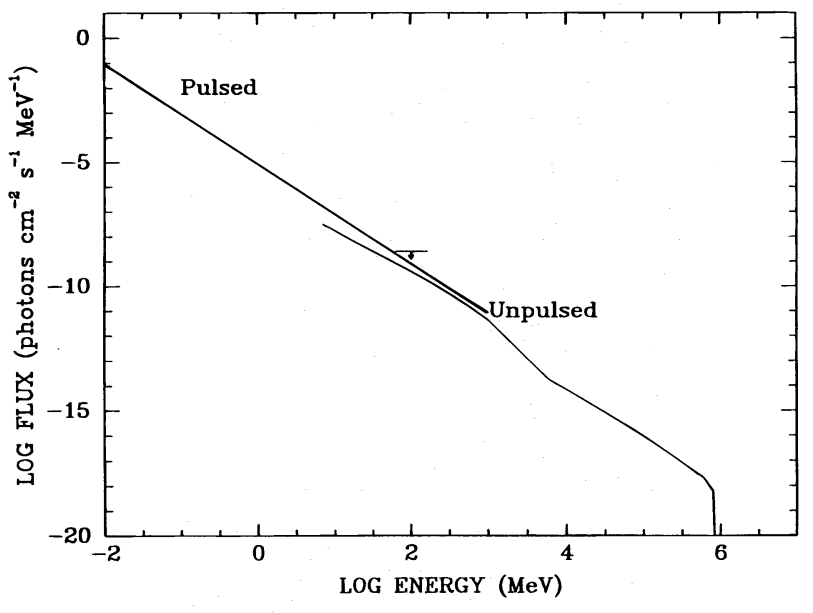

Fig. 4.- The calculated pulsed and unpulsed gamma-ray spectra of PSR J0751+18. The downward arrow is the observed upper limit by EGRET at $E_{\text {crit }} \sim 1.2 \mathrm{GeV}$. 


\section{REFERENCES}

Bhattacharya, D., \& van den Heuvel, E. P. J. 1991, Phys. Rep., 203, 1

I Blumenthal, G. R., \& Gould, R. J. 1970, Rev. Mod. Phys., 42, 237

Chen, K., \& Ruderman, M. 1993, ApJ, 402, 624

Cheng, K. S., \& Ding, W. K. Y. 1994, ApJ, 431, 724

Cheng, K. S., Ho, C., \& Ruderman, M. 1986a, ApJ, 300, 500 (CHR I)

In $1986 \mathrm{~b}$, ApJ, 300, 522 (CHR II)

III Cheng, K. S., \& Wei, D. M. 1995, ApJ, 448, 281

'-I Cheung, W. M., \& Cheng, K. S. 1993, ApJ, 413, 694

Fichtel, C. E., et al. 1993, A\&A, 94, 13

Gould, R. J. 1965, Phys. Rev. Lett., 15, 577

Grindlay, J. E., \& Hoffman, J. A. 1971, Astrophys. Lett., 8,209

Hartman, R. C., et al. 1992, BAAS, 24, 1155

Ho, C. 1989, ApJ, 342, 396
Jauch, J. M., \& Rohrlich, F. 1976, The Theory of Photons and Electrons (2d ed.; New York: Springer)

Kulkarni, S. R., Narayan, R., \& Romani, R. W. 1990, ApJ, 356, 174

Kwok, P. W., Cheng, K. S., \& Lau, M. M. 1991, ApJ, 379, 653

Lundgren, S. C., Zepka, A. F., \& Cordes, J. M. 1993, IAU Circ. 5878 1995, ApJ, 453, 419

Manchester, R. N., et al. 1991, Nature, 352, 219

Michelson, D. L., et al. 1994, ApJ, 435, 218

Rieke, G. H., \& Weekes, T. C. 1969, ApJ, 155, 429

Sturner, S. J., \& Dermer, C. D. 1994, A\&A, 281,L101

Tavani, M. 1993, ApJ, 407, 135

Wijers, R., \& Van Paradijs, J. 1991, A\&A, 241,L37 